\title{
Validation of a New Semi-Automated Technique to Evaluate Muscle Capillarization
}

\author{
Sam B. Ballak ${ }^{1,2}$, Moi Hoon Yap ${ }^{1}$, Peter J. Harding1, Hans Degens ${ }^{1}$
}

\author{
${ }^{1}$ School of Healthcare Science, Cognitive Motor Function Research Group, Manchester \\ Metropolitan University, Manchester, United Kingdom. \\ ${ }^{2}$ Laboratory for Myology, MOVE Research Institute Amsterdam, Faculty of Human \\ Movement Sciences, VU University Amsterdam, Amsterdam, The Netherlands
}

\begin{abstract}
To study capillarization of skeletal and heart muscle the method of capillary domains has often been used. However, the conventional data processing method using a digitizing tablet is an arduous task and time consuming. Here we compare a new semi-automated capillary domain data collection and analysis in muscle tissue with the standard capillary domain method. The capillary density $\left(1481 \pm 59\right.$ vs. $1447 \pm 54$ caps $\left.\left.\cdot \mathrm{mm}^{-2}\right) ; \mathrm{R}^{2}: 0.99 ; P<0.01\right)$ and heterogeneity of capillary spacing $\left(0.0848 \pm 0.002\right.$ vs. $\left.0.085 \pm 0.002 ; \mathrm{R}^{2}: 0.95 ; P<0.01\right)$ were similar in both methods. Although the fiber cross-sectional area was $\sim 8 \%$ larger $(P=$ $0.08)$ in the old than new method, but correlated well $\left(\mathrm{R}^{2}: 0.84 ; P<0.01\right)$. This was likely due to differences in outlining the contours between the two methods. In conclusion, the semi-automated method gives quantitatively and qualitatively similar data as the conventional method and saves a considerable amount of time. In addition, this new method has the option to add more parameters.
\end{abstract}

\section{Introduction}

An adequate blood supply to the muscle tissue is important not only for delivery of oxygen to the working muscle, but also for the removal of metabolites and heat. The exchange between the blood and muscle tissue takes place in the capillaries and an adequate muscle capillarization is thus crucial for muscle function. The capillary supply to a fiber is determined by its size, type, mitochondrial content and metabolic surrounding. During hypoxia, hypertrophy and maturational growth capillary proliferation occurs to maintain an adequate muscle oxygenation as far as possible. 
The method of capillary domains has been used previously to study the capillarization in skeletal and heart muscle. The strengths of the method are that it not only provides measures of overall capillary supply, such as the capillary density (CD in caps $\cdot \mathrm{mm}^{-2}$ ) and capillary to fiber ratio, but also the capillary supply to individual fibers. It is also unique in that it gives an indication of the heterogeneity of capillary spacing, which can have a significant impact on muscle oxygenation and is an accurate method to estimate the oxygen supply areas of individual capillaries and indirect indicator of tissue oxygenation. The data obtained with the method of capillary domain can be fed into models of tissue oxygenation . The drawback of the method is, however, that data collection is a manual and time-consuming process. First pictures have to be printed, fibers and capillaries manually traced on paper and then traced again on a digitizer. The coordinates of the capillaries and fiber outlines are then processed and analyzed with AnaTis (BaLoH Software, www.baloh.nl) .

Automation would significantly reduce the data processing time, potentially reduce human errors and improve the accuracy of the data processing. A semiautomated method would also provide the possibility to expand the analysis by introducing new parameters.

We developed a semi-automated system using Matlab R2012a ${ }^{\circledR}$ where the computer-based manual annotation tools were developed to annotate the border coordinates, capillary coordinates, and the coordinates of the fiber outlines. Subsequently an automated program was developed to analyze the results from the manual annotation. To compare and validate the results produced by our semiautomatic method with the conventional method, ten $m$. plantaris sections of 9month-old mice were analyzed. Thereto, the correlations and differences between both methods were assessed.

\section{Methods}

\section{Sampling}

The left $m$. plantaris of ten 9-month-old C57BL/6j mice were excised, frozen in liquid nitrogen at optimal length and stored at $-80^{\circ} \mathrm{C}$ for further analysis.

\section{Immunohistochemistry}

Sections of $10 \mu \mathrm{m}$ were cut in a cryostat at $-20^{\circ} \mathrm{C}$ and stained with biotinylated lectin (Griffonia simplicifolia) to locate capillaries. Sections were fixed in ice-cold acetone for $15 \mathrm{~min}$ and washed with HEPES-buffer for $5 \mathrm{~min}$. After blocking with $0.1 \%$ BSA in HEPES for 60 min the sections were washed with HEPES again and incubated with peroxide for $30 \mathrm{~min}$. Subsequently, the sections were washed with HEPES for $5 \mathrm{~min}$, incubated with the lectin for an hour (Vector Laboratories, UK) and three times washed with HEPES for $5 \mathrm{~min}$. After incubation with VIB substrate kit (Vector Laboratories, UK), the sections were washed with $\mathrm{dH}_{2} \mathrm{O}$ for 5 min before enclosing with glycerin-gelatine. 


\section{Old software for analysis of capillarization}

The capillarization was analyzed first as described previously . In short, the coordinates of the capillaries and the outlines of the muscle fibers were manually delineated and digitized with a digitizing tablet (Summagraphics MM1201) and the data fed into AnaTis (BaLoH Software, www.baloh.nl) to calculate capillary domains. Domains are areas surrounding a capillary delineated from surrounding capillaries by equidistant boundaries. It also gives an index of the heterogeneity of capillary spacing as the logarithmic standard deviation of the domain areas $\left(\log _{R} S D\right)$ and overall indices of capillarization, such as capillary density $(C D$; cap. $\mathrm{mm}^{-2}$ ). In addition, the program calculates the fiber cross-sectional areas (FCSA) and provides indices of the capillary supply to individual fibers: the local capillary to fiber (LCFR) is calculated as the sum of the fractions of the capillary domains overlapping a given fiber and the capillary fiber density (CFD; cap $\cdot \mathrm{mm}^{-2}$ of a given fiber) is the LCFR divided by the FCSA of that fiber.

\section{Semi-automated software for analysis of capillarization}

We developed a semi-automated program for capillary domain analysis. Two annotation tools were developed using Matlab ${ }^{\circledR}$ libraries. The first annotation tool was used to select the capillary and border coordinates. The second annotation tool delineated the fiber boundaries. Subsequently, a data analysis function was implemented in which the capillary domain was calculated (via voronoi tessellations). Where a capillary domain or a fiber outline crossed the border, it would be considered a border domain or fiber. All areas and perimeters were calculated by custom Matlab ${ }^{\circledR}$ functions, and all statistics and metrics were compiled from these data.

Thus the CD, the FCSA of both complete and incomplete (those crossing the border) fibers was calculated as well as the LCFR and CFD.

\section{Statistical analysis}

The data of the two methods was compared with a paired Student $t$ test and correlations $\left(\mathrm{R}^{2}\right)$. Data are presented as mean \pm standard error of the mean (SEM). A value of $P<0.05$ was considered significant.

\section{Results}

Figure 1A shows a comparison of the $\mathrm{CD}$ obtained with the conventional and new method. The line in the graph represents the line of identity. The CD in the conventional and new method (1481 \pm 59 vs. $1447 \pm 54$ cap $\left.\cdot \mathrm{mm}^{-2}\right)$ are highly correlated $\left(\mathrm{R}^{2}: 0.99, P<0.01\right)$. Figure 1B shows the heterogeneity of capillary spacing $\left(\log _{R} S D\right)$ for the conventional and new method $(0.0848 \pm 0.002$ vs. $0.0850 \pm 0.002)$. The $\log _{\mathrm{R}} \mathrm{SD}$ for both methods are strongly correlated $\left(\mathrm{R}^{2}: 0.95, P\right.$ 
$<0.01)$. The correlation for the FCSA between the two methods is strong $\left(\mathrm{R}^{2}\right.$ : $0.87, P<0.01$; Fig. $1 C$ ) but the new method gives consistently lower FCSAs than the old method the FCSA in the new method $\left(1693 \pm 66\right.$ vs. $1531 \pm 55 \mu \mathrm{m}^{2} ; P=$ $0.08)$.

Table 1 shows that the percentage of connective tissue (\%CT) did not correlate very strongly between the two methods. Furthermore, although the LCFR and the CFD correlated strongly ( $\mathrm{R}^{2}: 0.80$ and $0.86 ; P<0.01$ ), the new method gave consistently lower values for both LCFR and CFD than the old method $(P<0.01$ and $P=0.01$ ).

\section{Discussion}

The most important outcome of this study is that a newly developed Matlab (B based semi-automatic method of capillary domains provides quantitatively and qualitatively the same outcome for parameters of overall capillary supply as the traditional manual method. Although the FCSA is underestimated in the new method, affecting quantitatively also indices of the capillary supply to individual fibers, this is a systematic underestimate. Therefore. the qualitative outcome is similar for the two methods and the newly developed method can be readily used for comparative studies on changes in muscle or cardiac capillary supply.

The $C D$ and $\log _{R}$ SD obtained by the two methods were virtually identical. This is indicates that the coordinates recorded for each capillary were comparable between the methods. It also indicates that the calculation of the capillary domains is comparable between the two methods as for the calculation of the $\log _{R} S D$ the surface areas of the individual domains and the radius of circles with corresponding surface areas has to be calculated.

There was, however, a small difference in the way the fiber outlines were traced. In the conventional method, accidental fiber overlap could be accounted for in the old method, while in the Matlab ${ }^{\circledR}$ based software, fibers overlapping each other are joined and seen as one big fiber. Therefore, even with good tracing skills a researcher may avoid tracking the borders too close to each other to prevent fusion of two separate fibers in the subsequent analysis as one fiber; in other words the experimenter may intentionally draw the fibers slightly too small, resulting in a smaller fiber area. However, the conventional method of tracing the fiber boundaries, could lead to a small overestimation of the size of the fibers, since overlapping areas will be counted twice. Another difference is that the conventional method traced the coordinates of the boundaries continuously, allowing insertion of small human errors. In the new method, however, the user can click and include the exact coordinates he wants. Also, it is much easier to delete incorrect coordinates. Together, this stresses the importance of good tracing skills in both the new and the old method. 
The difference in fiber size, caused by the difference in tracing the outlines of the fibers also has an impact on the \% CT, which was significantly higher in the new method. The differences in fiber size also work through in the LCFR and CFD, which were lower in the new than the old method, even though the correlation between the two methods was strong. It can be seen that the FCSA was systematically underestimated in the new method. Thus, while there is a systematic qualitative difference between the two methods, the new method is readily applicable in comparative studies.

We are currently working on the tracing algorithm to adjust the systematic underestimation of the FCSA in the Matlab ${ }^{\circledR}$ based new software. The aim of this study was primarily to evaluate the validity of this technique before looking into that issue.

The new Matlab (B) based program saves on average about 1.5 hour per section/photo. This will increase even more when additional (parts) of the analysis are automated further. In addition, the Matlab ${ }^{\circledR}$ based program makes analysis more flexible and allows the implementation of new variables in the future. This new time-sparing method is the first step in fully automating the process and assessment of capillary domains. Future research should focus on fully automating the sampling of capillary and fiber outline coordinates.

\section{Conclusion}

In conclusion, the new semi-automated Matlab (B) based method is highly comparable to the standard method of capillary domain analysis when considering indices of overall muscle capillarization. However, due to differences in the way of tracing the fiber outlines there are small difference in FCSA, which also affects indices of capillary supply to individual fibers. This is currently under further development. Together, this new method appears be a valid way to qualitatively and quantitatively analyze the capillarization in cardiac and skeletal muscle.

Acknowledgments This research was funded by the European Commission through MOVEAGE, an Erasmus Mundus Joint Doctorate program (2011-2015).

\section{References}

1. Ahmed SK, Egginton S, Jakeman PM, et al. 1997 Is human skeletal muscle capillary supply modelled according to fibre size or fibre type? Exp Physiol.82(1):231-4

2. Degens H, Turek Z, Hoofd LJ, et al. 1992 The relationship between capillarisation and fibre types during compensatory hypertrophy of the plantaris muscle in the rat. $\mathrm{J}$ Anat.180 ( Pt 3):455-63 
3. Wust RC, Gibbings SL, Degens H. 2009 Fiber capillary supply related to fiber size and oxidative capacity in human and rat skeletal muscle. Adv Exp Med Biol.645:75-80

4. Wust RC, Jaspers RT, van Heijst AF, et al. 2009 Region-specific adaptations in determinants of rat skeletal muscle oxygenation to chronic hypoxia. Am J Physiol Heart Circ Physiol.297(1):H364-74

5. Degens H, Deveci D, Botto-van Bemden A, et al. 2006 Maintenance of heterogeneity of capillary spacing is essential for adequate oxygenation in the soleus muscle of the growing rat. Microcirculation.13(6):467-76

6. Hoofd L, Turek Z, Kubat K, et al. 1985 Variability of intercapillary distance estimated on histological sections of rat heart. Adv Exp Med Biol.191:239-47

7. Degens H, Ringnalda BE, Hoofd LJ. 1994 Capillarisation, fibre types and myoglobin content of the dog gracilis muscle. Adv Exp Med Biol.361:533-9

8. Al-Shammari AA, Gaffney EA, Egginton S. 2012 Re-evaluating the use of Voronoi Tessellations in the assessment of oxygen supply from capillaries in muscle. Bull Math Biol.74(9):2204-31

9. Liu G, Mac Gabhann F, Popel AS. 2012 Effects of fiber type and size on the heterogeneity of oxygen distribution in exercising skeletal muscle. PLoS One.7(9):e44375

10. Al-Shammari AA, Gaffney EA, Egginton S. 2014 Modelling capillary oxygen supply capacity in mixed muscles: Capillary domains revisited. J Theor Biol.356c:47-61

11. Hoofd L, Degens H. 2013 Statistical treatment of oxygenation-related data in muscle tissue. Adv Exp Med Biol.789:137-42

Table 1. Correlations and $P$-values of indices of capillary supply to individual fibers (LCFR: local capillary to fiber ration; CFD: capillary fiber density) and \% connective tissue (\%CT) between the two methods in $m$. plantaris of 9-months-old male C57B1/6j mice.

\begin{tabular}{lllll}
\hline & Conventional method & New method & $\mathrm{R}^{2}$ & P-value \\
\hline \%CT $(\%)$ & $11.5 \pm 0.6$ & $16.7 \pm 1.4$ & 0.43 & 0.01 \\
LCFR & $2.503 \pm 0.076$ & $1.998 \pm 0.07$ & 0.80 & 0.00 \\
CFD $\left(\mathrm{mm}^{-2}\right)$ & $1564 \pm 56$ & $1252 \pm 46$ & 0.86 & 0.00 \\
\hline
\end{tabular}

1
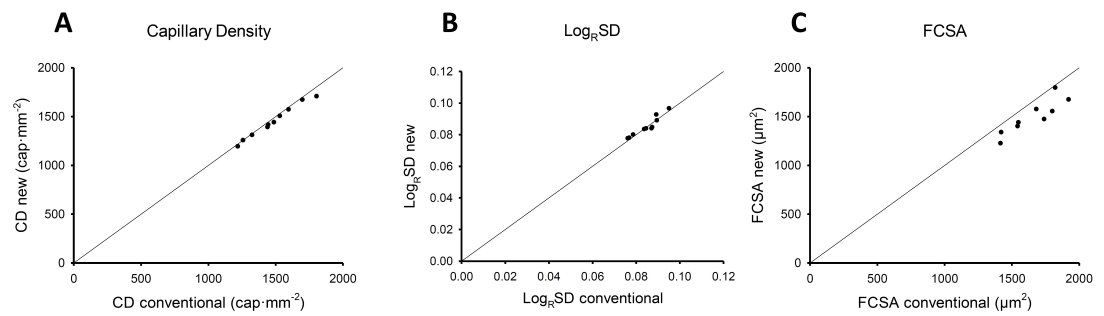

Fig 1. The correlation between the old and new method for Capillary density (cap $\left.\cdot \mathrm{mm}^{-2}\right)$ $\left(\mathrm{R}^{2}=0.99 ; P<0.01\right)(\mathrm{A}), \log _{\mathrm{R}} \mathrm{SD}\left(\mathrm{R}^{2}=0.95 ; P<0.01\right)(\mathrm{B})$ and FCSA $\left(\mu \mathrm{m}^{2}\right)\left(\mathrm{R}^{2}=0.87 ; P<0.01\right)$ (C) compared between the conventional $(x$-axis) and new method $(y$-axis). The line represents the line of identity. 